elucidated. However, it is clear that future research and policy initiatives to encourage families to prioritise sleep in the family routine has the potential to improve not only cognitive and academic outcomes but also child health and wellbeing.

\section{P064 ASSOCIATION BETWEEN SELF-REPORTED SLEEP- DISORDERED BREATHING AND ESTIMATED CARDIOVASCULAR RISK}

'Lyudmila Korostovtseva*, ${ }^{1}$ Oxana Rotar, ${ }^{1}$ Asiyat Alieva, ${ }^{1}$ Mikhail Bochkarev, ${ }^{1}$ Maria Boyarinova, ${ }^{1,2}$ Nadezhda Zvartau, ${ }^{1,3}$ Yurii Sviryaev, ${ }^{1,2}$ Aleksandra Konradi, ${ }^{1}$ Eugene Shlyakhto. ${ }^{1}$ Almazov National Medical Research Centre, St Petersburg, Russia; 2ITMO University, St Petersburg, Russia; ${ }^{3}$ Sechenov Institute of Evolutionary Physiology and Biochemistry of RAS, St Petersburg, Russia

10.1136/bmjresp-2019-bssconf.64

Background Sleep-disordered breathing (SDB) is associated with the increased cardiovascular (CV) morbidity, however, it is not considered in the standard risk stratification scales.

Purpose We evaluated the association between self-reported SDB (population-based sample) and two common risk stratification scales: SCORE and ASCVD.

Methods We selected 526 adults without known CV-disease (156 males, 30\%; mean age $54 \pm 6.8$ years) from the population-based sample (the epidemiological study ESSE-RF). All subjects were interviewed (lifestyle, medical history, complaints) using standard questionnaire. We assessed selfreported snore ('Do you snore?') and sleep apnea ('Do you have sleep apneas?'). Affirmative response was considered diagnostic. The 10-year risk of fatal CV-events was assessed by the SCORE high-risk charts calculator and ASCVD risk estimator.

Results Overall, 288 (55\%) subjects had snoring, while 27 $(5 \%)$ reported sleep apneas. Based on the SCORE the participants were divided as following: low risk $(<1 \%)$ was the most predominant category $(35 \%)$; moderate risk $(\geq 1 \%$ and $<5 \%)-44 \%$, high risk (5-10\%)-16\%, and very high risk-5\%. Based on the ASCVD scale the subjects were divided as following: low risk $(<5 \%)$ consisted 55,4\%; borderline (67,4\%)-16,3\%, intermediate (7,5-19,9\%)-23\%, and high-5,3\%. High-to-very-high SCORE-risk was more frequent in subjects with self-reported snoring compared to non-snorers: $27.1 \%$ vs. $7.5 \%$, respectively (Chi-square $=44.5, \mathrm{p}<0.001$ ). No association was found between self-reported sleep apneas and CVrisk $(p>0.05)$. Similarly, intermediate-high ASCVD-risk is found more often in snorers vs. non-snorers $(34.7 \%$ vs. $16.4 \%$, respectively, Chi-square $=22.5, \mathrm{p}<0.001$ ), with no association between self-reported sleep apneas and ASCVD-risk $(p>0.05)$. Logistic multiple regression demonstrated an association between SCORE-risk and self-reported snoring $(\mathrm{OR}=3.2195 \% \mathrm{CI} 1.82-5.67, \mathrm{p}<0.001)$; between self-reported snoring and ASCVD-risk $(\mathrm{OR}=2.24 \quad 95 \% \mathrm{CI}$ 1.35-3.71, $\mathrm{p}=0.002$ ).

Conclusions In Russian population-based sample self-reported snoring (unlike self-reported sleep apnea) is associated with the increased 10-year risk of CV-events independently of the risk stratification scale. The lack of association between CVrisk and sleep apnea might be related to the subjective assessment.

(grant\#HШ-5508.2018.7)

\section{P065} SLEEP-WAKE CYCLE AND MELATONIN LEVELS IN PATIENTS WITH DISORDERS OF CONSCIOUSNESS

${ }^{1}$ Ekaterina Kondratieva, 'Lyudmila Korostovtseva*, ${ }^{1}$ Ivan Ternovykh, ${ }^{1}$ Mikhail Bochkarev, ${ }^{1}$ Sergey Kondratiev, ${ }^{3}$ Maria Frolova, ${ }^{1}$ Tatiana Alekseeva, ${ }^{1,2} Y$ urii Sviryaev, ${ }^{3}$ Natalia Zybina, ${ }^{3}$ Natalia Dryagina, ${ }^{1}$ Anatolii Kondratiev. ${ }^{1}$ Almazov National Medical Research Centre, St Petersburg, Russia; ${ }^{2}$ Sechenov Institute of Evolutionary Physiology and Biochemistry of RAS, St Petersburg, Russia; ${ }^{3}$ The Nikiforov Russian Center of Emergency and Radiation Medicine, St Petersburg, Russia

\subsection{6/bmjresp-2019-bssconf.65}

Background and aims Sleep is considered to play an important role in neuroplasticity and posttraumatic brain recovery. The assessment of sleep structure and sleep-wake cycle in subjects with disorders of consciousness (DOC) might be useful for predicting prognosis and treatment approach modification. In our study we assessed sleep structure and sleep-wake cycle by long-term $(\geq 24 \mathrm{~h})$ polysomnography (PSG), melatonin and stress hormones levels in DOC patients - unresponsive wakefulness syndrome (UWS) and minimally conscious state (MCS). Methods We included 27 patients (22-57 years old). Median time duration after the brain injury constituted 11 (1-96) months. We applied Coma Recovery Scale (CRS-R) for behavioral assessment and diagnosed UWS in 7 subjects and MCS in 20 patients (traumatic brain injury was the underlying reason in 20 subjects, hypoxia - in 5, other causes were verified in 2 cases). The full in-hospital attended PSG (Medicom, Russia) was recorded during 24 hours. Blood sampling for melatonin were evaluated 5 times a day $(8.00,15.00,21.00$, $00.00,3.00)$, urine samples for melatonin were taken twice (day and night).

Results All (100\%) patients demonstrated irregular sleep-wake cycle and fragmented, abnormal sleep structure, while $85 \%$ patients had normal melatonin level (within the reference values). Deep sleep was absent in $25 \%$ cases. REM sleep episodes, despite decreased duration, were present in the majority of patients (87\%). There was no significant correlation between melatonin level and PSG parameters.

Conclusion In our study, DOC patients had irregular sleepwake cycle and sleep structure violations. However, sleep-wake changes do not seem to be associated with the disorders of melatonin metabolism.

(the study is supported by the grant of the RFFR \#19-2901066)

\section{P066 THE ASSOCIATION BETWEEN THE LEVELS OF BRAIN- DERIVED NEUROTROPHIC FACTOR AND STRUCTURAL AND FUNCTIONAL PARAMETERS OF THE BRAIN IN CHRONIC INSOMNIA VS HEALTHY SUBJECTS}

${ }^{1}$ Lyudmila Korostovtseva*, ${ }^{1}$ Irina Filchenko, ${ }^{1}$ Mikhail Bochkarev, ${ }^{1}$ Elena Medvedeva, ${ }^{1}$ Eugeniya Kazakova, ${ }^{1}$ Elena Vasilieva, ${ }^{1,2}$ Yurii Sviryaev, ${ }^{1}$ Mikhail Zykov, ${ }^{1}$ Darya Ryzhkova. ${ }^{1}$ Almazov National Medical Research Centre, St Petersburg, Russia; ${ }^{2}$ Sechenov Institute of Evolutionary Physiology and Biochemistry of RAS, St Petersburg, Russia

\subsection{6/bmjresp-2019-bssconf.66}

Background Brain-derived neurotrophic factor (BDNF) plays a crucial role in the neuronal networking and reorganization. BDNF levels are changed in neurological and psychiatric diseases. We assessed BDNF levels in patients with chronic primary insomnia (PI) and their association with the brain metabolism. 